\title{
CATALOG OF THE FAMILY CALLIPHORIDAE (DIPTERA: OESTROIDEA) OF MEXICO
}

\author{
CATÁLOGO DE LA FAMILIA CALLIPHORIDAE (DIPTERA: OESTROIDEA) DE MÉXICO
}

\author{
SANTIAGo JAUME-SCHINKEL, SERgIO IBÁÑEZ-BERNAL** \\ Red Ambiente y Sustentabilidad, Instituto de Ecología, A.C. (INECOL), Carretera antigua a Coatepec 351, Colonia El Haya, \\ Xalapa, Veracruz, México. C.P. 91073.<santijaumes@hotmail.com>; <sergio.ibanez@inecol.mx> \\ *Corresponding author: 〈sergio.ibanez@inecol.mx>
}

Received: 09/09/2019; accepted: 15/01/2020; published online: 11/02/2020

Responsible editor: Karina D. Rivera-García
Jaume-Schinkel, S., Ibáñez-Bernal, S. (2020) Catalog of the family Calliphoridae (Diptera: Oestroidea) of Mexico. Acta Zoológica Mexicana (nueva serie), 36, 1-25. https://doi.org/10.21829/azm.2020.3612237

\begin{abstract}
Calliphoridae is a worldwide distributed family of flies with over 1,500 described species. There are approximately 100 species in the Nearctic region and 130 in the Neotropical region, but in the Mexican territory only few species have been recorded. There is not a previous catalog of Mexican calliphorids. This catalog summarizes taxonomic actualized information of 30 species of 11 genera and three subfamilies of Calliphoridae known up to date in Mexico.
\end{abstract}

Key words: blow flies; taxonomy; species list

Jaume-Schinkel, S., Ibáñez-Bernal, S. (2020) Catálogo de la familia Calliphoridae (Diptera: Oestroidea) de México. Acta Zoológica Mexicana (nueva serie), 36, 1-25. https://doi.org/10.21829/azm.2020.3612237

RESUMEN. La familia Calliphoridae está presente en casi todo el mundo con más de 1,500 especies descritas. Existen aproximadamente 100 especies en la región Neártica y 130 en la región Neotropical, pero en el territorio mexicano solo pocas especies se han registrado. No existe un catálogo previo de califóridos de México. Este catálogo recopila la información taxonómica básica y actualizada de las 30 especies de 11 géneros y tres subfamilias de Calliphoridae conocidos hasta el momento en México.

Palabras clave: moscas metálicas; taxonomía; lista de especies

\section{INTRODUCTION}

Flies of the family Calliphoridae are commonly known in English as blow flies, green bottles or blue bottles, and in Spanish "moscas metálicas" or "moscas panteoneras", with some species very familiar to people around the world (Prado e Castro et al., 2016). It has a great diversity within calyptrate Diptera with about 1,500 described species belonging to 150 genera present in all biogeographic regions (Rognes, 1991). 
Blow flies are medium-size Diptera (4-16 mm) some with a beautiful metallic color (iridescence) ranging from green, blue to purplish and violet, while some others have duller coloration, but all with some degree of metallic reflections. Males are usually holoptic and females dichoptic with chaetotaxy similar in both sexes (Prado e Castro et al., 2016; Wolff \& Kosmann, 2016). Adult Calliphoridae are found in wide variety of habits; some species feed on nectar and are pollinators, other species are parasites of worms, bird nestlings and snails, a few have medical and veterinary importance causing myiasis or mechanical transmission of pathogens, but the bulk of species are associated with decaying animal and vegetable matter and feces (Norris, 1966; Rognes, 1998; Zumpt, 1965; Prado e Castro et al., 2016). Immature stages feed on a wide variety of substrates including carcasses and healthy/necrotic tissues of vertebrates, and some feed on amphibians, birds or mammals' blood (Wolff \& Kosmann, 2016). These habits allow the family to play an important ecological key role as decomposers, being a useful tool in forensic entomology studies for that reason (Byrd \& Castner, 2010). Blow fly species show different levels of synanthropy (association with human habitats), some associated with high disturbed habitats as urban environments and human settlements (eusynanthropy), modify ecosystems for agricultural, pastoral and forestry practices (hemisynanthropy) or natural pristine ecosystems (asynanthropy) (Povolnỳ, 1971).

Phylogeny of Calliphoridae is still debatable and some authors believe it is not a monophyletic group (Rognes, 1997; Kutty et al., 2010). Some subfamilies of Calliphoridae have been recently elevated to the family status based in morphological and molecular data, such as Mesembrinellidae and Rhiniidae (Kutty et al., 2010; Marinho et al., 2012, 2017; Cerretti et al., 2017), changes that we follow in this work. Some authors have contributed since 1960's with a variety of studies regarding the blow fly fauna in the Neotropical region (James, 1955, 1966, 1970, 1971; Mello, 1961, 1962, 1965, 1967, 1968, 1969a, 1969b, 1972a, 1972b, 1974, 1978, 1996, 2003; Whitworth, 2014; Wolff, 2013) and some others to the Nearctic region fauna (e.g. Hall, 1948; Tantawi \& Whitworth, 2014; Tantawi et al., 2017; Whitworth, 2006).

The number of species distributed in America is significantly smaller as compared to the rest of the world (Wolff \& Kosmann, 2016), as there are nearly 100 species in the Nearctic region (Pape et al., 2009) and 130 species in the Neotropical region (Pape et al., 2011). Considering that Mexico belongs to the biogeographic transition zone between Nearctic and Neotropical areas (Halffter, 2003), with a variety of ecosystems, the number of species expected in Mexico should be higher than the number known to date. This is probably consequence of the scarcity of faunistic studies on this group.

There are few catalogs of American Calliphoridae and no one specific for Mexico. The Catalog of the Diptera of America North of Mexico (Hall, 1965), presented species distributed in North America with few records in Mexico which unfortunately is not updated taxonomically, whereas the Catalogue of the Diptera of the Americas south of the United States (James, 1970) do not include all the Mexican species known today, also in a non-updated classification. Some South and Central America catalogs have been published recently in different countries such as Chile (Mac-Lean \& González, 2006), Nicaragua (Maes et al., 1994), Colombia (Wolff \& Kosmann, 2016), and Systema Dipterorum available online, with records on nomenclature and references of several worldwide species (Pape \& Evenhuis, 2019), and for Mexico there is one catalog of Diptera available online at CONABIO (Ibáñez-Bernal, 2017), which includes Mesembrinellidae as subfamily and other species as valid that now are synonyms.

In the particular case of Mexico, studies regarding this family are few and disperse, some are focused on the entomological succession on corpses (Byrd \& Castner, 2010; Flores, 2009; García-Espinoza et al., 2012; Mañas-Jordá et al., 2017; Stanford-Camargo et al., 2017; Valdés-Perezgasga et al., 2012). Nevertheless, scarcity of systematized and periodic studies in nearly all the Mexican territory makes difficult to present a complete list of Mexican species, moreover, to trace the geographic distribution of species.

In this work an up-to-date catalog of Calliphoridae (Diptera) for Mexico based on an extensive literature review is presented. It includes 30 species, in 11 genera and three subfamilies (Table 1), with their 
synonyms and early name combinations and references. Information is presented by category starting with family, genus and species, followed by the author (s) and year of publication of the original description. The original reference, type species for supraspecific taxa and type locality for species are also presented. A chronological list of synonyms for each taxon, with the author (s), year of publication, starting page of mention and type specimens for supraspecific taxa and type locality for species, are provided. For each species the known distribution by country is given and in the case of Mexican records the states in which the species are known are presented between parentheses. For some species we present notes or additional useful references that we consider to be complemental information regarding taxonomical notes or further discussions.

Table 1. Calliphoridae species recorded in Mexico.

\begin{tabular}{|c|c|c|c|}
\hline Subfamily & Genus & Species & Author \\
\hline \multirow{10}{*}{ Calliphorinae } & \multirow{6}{*}{ Calliphora } & coloradensis & Hough, 1889 \\
\hline & & grahami & Aldrich, 1930 \\
\hline & & irazuana & Townsend, 1908 \\
\hline & & latifrons & Hough, 1889 \\
\hline & & triseta & Whitworth, 2012 \\
\hline & & vicina & Robineau-Desvoidy, 1830 \\
\hline & Cynomya & cadaverina & Robineau-Desvoidy, 1830 \\
\hline & \multirow{3}{*}{ Protocalliphora } & asiovora & Shannon \& Dobroscky, 1924 \\
\hline & & beameri & Sabrosky, Bennett \& Whitworth, 1989 \\
\hline & & sialia & Shannon \& Dobroscky, 1924 \\
\hline \multirow{8}{*}{ Lucilinae } & \multirow{8}{*}{ Lucilia } & cluvia & (Walker, 1849) \\
\hline & & cuprina & (Wiedemann, 1830) \\
\hline & & eximia & (Wiedemann, 1830) \\
\hline & & illustris & (Meigen, 1826) \\
\hline & & mexicana & Macquart, 1843 \\
\hline & & purpurascens & (Walker, 1836) \\
\hline & & sericata & (Meigen, 1826) \\
\hline & & silvarum & (Meigen, 1826) \\
\hline \multirow{9}{*}{ Chrysomyinae } & Cochliomyia & $\begin{array}{l}\text { hominivorax } \\
\text { macellaria }\end{array}$ & $\begin{array}{l}\text { (Coquerel, 1858) } \\
\text { (Fabricius, 1775) }\end{array}$ \\
\hline & \multirow{3}{*}{ Compsomyiops } & callipes & (Bigot, 1877) \\
\hline & & fulvicrura & (Robineau-Desvoidy, 1830) \\
\hline & & melloi & Dear, 1985 \\
\hline & Chloroprocta & idioidea & (Robineau-Desvoidy, 1830) \\
\hline & Chrysomya & $\begin{array}{l}\text { megacephala } \\
\text { rufifacies }\end{array}$ & $\begin{array}{l}\text { (Fabricius, 1794) } \\
\text { (Macquart, 1843) }\end{array}$ \\
\hline & Hemilucilia & $\begin{array}{l}\text { segmentaria } \\
\text { semidiaphana }\end{array}$ & $\begin{array}{l}\text { (Fabricius, 1805) } \\
\text { (Rondani, 1850) }\end{array}$ \\
\hline & Paralucilia & fulvinota & (Bigot, 1877) \\
\hline & Phormia & regina & (Meigen, 1826) \\
\hline
\end{tabular}




\section{Family Calliphoridae Brauer \& Bergenstamm, 1889}

Calliphorinae Brauer \& Bergenstamm, 1889: 85. Type: Calliphora Robineau-Desvoidy.

Additional references: Sabrosky, 1999: 85.

\section{Subfamily Calliphorinae Brauer \& Bergenstamm, 1889 Genus Calliphora Robineau-Desvoidy, 1830}

Calliphora Robineau-Desvoidy, 1830: 433. Type species: Musca vomitoria Linnaeus, 1758: 595.

Steringomyia Pokorny, 1889: 568. Type species: Steringomyia stylifera Pokorny, 1889: 569.

Abonesia Villeneuve, 1927: 357. Type species: Musca genarum Zetterstedt, 1838: 658.

Acrophaga Brauer \& Bergenstamm, 1891: 367. Type species: Acophaga stelviana Brauer \& Bergenstamm, 1891: 367; Brauer, 1893: 500.

Eucalliphora Townsend, 1908: 118. Type species: Calliphora latifrons Hough, 1899a: 286.

Aldrichiella Rohdendorf, 1931: 177. Type species: Calliphora grahami Aldrich, 1930: 1. Junior homonym of Aldrichiella Vaughan, 1903: 101, and Aldrichiella Hendel, 1911: 37.

Stobbeola Enderlein, 1933: 126. Type species: Stobbeola norwegica Enderlein, 1933: 126.

Aldrichina Townsend, 1934a: 111. Type species: Calliphora grahami Aldrich, 1930: 1, replacement name for Aldrichiella Rohdendorf, 1931: 177.

Acronesia Hall, 1948: 272. Type species: Steringomyia aldrichia Shannon, 1923: 112.

\section{Calliphora coloradensis Hough, 1899}

Calliphora coloradensis Hough, 1899a: 286. Type locality: USA, Colorado.

Additional references: Shannon, 1923: 109, 1926: 135; Hall, 1948: 294; James, 1953: 143; Poole, 1996: 77; Whitworth, 2006: 699; Tantawi et al., 2017: 314.

Distribution: Canada, United States of America. Mexico (Durango, Morelos, Puebla) (Tantawi et al., 2017).

\section{Calliphora grahami Aldrich, 1930}

Calliphora grahami Aldrich, 1930: 1. Type locality: China, Sichuan Province.

Aldrichina grahami (Aldrich): Hall, 1948: 290.

Additional references: Thomas, 1951: 181; James, 1953: 144, 1955: 10; Hall, 1965: 929; Poole, 1996: 77; Whitworth, 2006: 699; Tantawi et al., 2017: 316.

Distribution: Mexico (Ciudad de México, Durango, Hidalgo) (Tantawi et al., 2017). This species is indigenous to the west Palearctic and Oriental regions but was introduced to Hawaii and Western of United States of America (Whitworth, 2006) and then to Mexico.

\section{Calliphora irazuana Townsend, 1908}

Calliphora irazuana Townsend, 1908: 118. Type locality: Costa Rica, Irazú.

Distribution: Costa Rica, El Salvador, Guatemala. Mexico (Chiapas, Ciudad de México, Durango, Estado de México, Guerrero, Puebla, Veracruz) (Whitworth, 2012). 


\section{Calliphora latifrons Hough, 1899}

Calliphora latifrons Hough, 1899a: 286. Type locality: USA, Idaho, Moscow.

Eucalliphora latifrons (Hough): Townsend, 1908: 118.

Eucalliphora arta Hall, 1948: 287. Type locality: Mexico, San Luis Potosí.

Calliphora arta (Hall): Whitworth, 2006: 698.

Calliphora bezzi Zumpt, 1956: 16. Type locality: Ruta, Italy.

Additional references: Shannon, 1923: 109; James, 1970: 12; Whitworth, 2006: 703; Rognes, 2016: 195; Tantawi et al., 2017: 326.

Distribution: Canada, United States of America. Mexico (Baja California, Chiapas, Ciudad de México, Durango, Puebla, Tabasco) (Tantawi et al., 2017). Tantawi et al. (2017) presented this species as indigenous of North America, but Rognes (2016) reported this species in China and Italy as Calliphora bezzi Zumpt, and he stated that apparently this species was dispersed by human activity, as it has only been found at international ports, leading to believe that the species has not stablished outside North America, but it can become a stablished population in the future.

\section{Calliphora triseta Whitworth, 2012}

Calliphora triseta Whitworth, 2012: 18. Type locality: Costa Rica, San Jose Province.

Additional references: Whitworth, 2012: 18.

Distribution: Costa Rica, El Salvador. Mexico (Chiapas, Oaxaca) (Whitworth, 2012). Its distribution overlaps with Calliphora irazuana, but Whitworth (2012) makes a clear difference to recognize both species, stating that this species may distribute in high altitudes of mountain areas thorough Central America.

\section{Calliphora vicina Robineau-Desvoidy, 1830}

Calliphora vicina Robineau-Desvoidy, 1830: 435. Type locality: USA, Philadelphia.

Musca carnivora Fabricius, 1794: 313. Name suppressed, see International Commission on Zoological Nomenclature (1992 [Opinion 1670]: 90).

Musca erythrocephala Meigen, 1826: 62. Junior primary homonym of Musca erythrocephala De Geer, 1776. Name suppressed, see International Commission on Zoological Nomenclature (1992 [Opinion 1670]: 90).

Calliphora littoralis Robineau-Desvoidy, 1830: 435. Type locality: Not specified.

Calliphora spitzbergensis Robineau-Desvoidy, 1830: 435. Type locality: Norway, Spitzbergen.

Calliphora monspeliaca Robineau-Desvoidy, 1830: 436. Type locality: France, Montpellier.

Calliphora musca Robineau-Desvoidy, 1830: 436. Type locality: Not specified.

Calliphora nana Robineau-Desvoidy, 1830: 436. Type locality: France, Paris.

Calliphora scutellata Macquart, 1834: 161. Type locality: France, Lille.

Musca thuscia Walker, 1849: 897. Type locality: USA, New York.

Calliphora rufifacies Macquart, 1851: 216. Type locality: USA, New York.

Musca aucta Walker, 1853: 334. Type locality: India, Madras or Calcutta.

Calliphora insidiosa Robineau-Desvoidy, 1853: 334. Type locality: France, Paris.

Calliphora turanica Rohdendorf, 1926: 90. Type locality: Not specified.

Additional references: Rognes, 1991: 63; Tantawi et al., 2017: 339. 
Distribution. This species is widespread around the world, mainly in large cities. In America it is present from Canada through Central and South America (Whitworth, 2006, 2012; Tantawi et al., 2017). In Mexico it has been recorded in Ciudad de México, but it is possible to be found in other states of Mexico. For detailed information see Rognes (1991).

\section{Genus Cynomya Robineau-Desvoidy, 1830}

Cynomya Robineau-Desvoidy, 1830: 363. Type species: Musca mortuorum Linnaeus, 1761: 452 (Macquart, 1834: 176).

Cynophaga Lioy, 1864: 890. Type species: Musca mortuorum Linnaeus.

Carcinomyia Townsend, 1915b: 21. Type species: Cynomya hirta Hough, 1898a: 166 (= Musca mortuorum).

Cynomyopsis Townsend, 1915c: 118. Type species: Cynomya cadaverina Robineau-Desvoidy, 1830: 365.

Cynomya cadaverina Robineau-Desvoidy, 1830

Cynomya cadaverina Robineau-Desvoidy, 1830: 365. Type locality: USA, Carolina.

Calliphora myoidea Robineau-Desvoidy, 1830: 436. Type locality: USA, Pennsylvania.

Calliphora aurulans Robineau-Desvoidy, 1830: 437. Type locality: USA, Carolina.

Calliphora compressa Robineau-Desvoidy, 1830: 438. Type locality: USA, Carolina.

Musca mortisequa Kirby, 1837: 316. Type locality: USA, Alaska.

Musca ilerda Walker, 1849: 895. Type locality: Canada, Ontario.

Cynomyia americana Hough, 1898b: 105. Type locality: USA, Louisiana.

Calliphora texensis Townsend, 1908: 116. Type locality: USA, Texas.

Cynomyopsis cadaverina (Robieneau-Desvoidy): Townsend, 1915a: 118.

Distribution: Alaska, Canada, Denmark, United States of America. Mexico (Aguascalientes) (Whitworth, 2006; Martínez-Ruvalcaba et al., 2009). Rognes (1991) mentioned this species to be widespread in the Nearctic and Palearctic regions, Hall (1948) mentioned it is a common species in United States of America and Canada, whereas Whitworth (2006) mentioned that he rarely found it in the southern United States. Martínez-Ruvalcaba et al. (2009) reported some specimens in the state of Aguascalientes, Mexico.

\section{Genus Protocalliphora Hough, 1899}

Protocalliphora Hough, 1899b: 65-66. Type species: Musca azurea Fallén, 1817: 245.

Avihospita Hendel, 1901: 29. Type species: Musca azurea Fallén, 1817: 245. Aldrich, 1901: 68; Aldrich, 1905: 523.

Apaulina Hall, 1948: 179. Type species: Protocalliphora avium Shannon \& Dobroscky, 1924: 249. Hamerstrom \& Hamerstrom, 1954: 5; James, 1955: 24; Zumpt, 1956: 94.

Trypocalliphora Peus, 1960: 199. Type species: Avihospita braueri Hendel, 1901: 30.

Additional references: Rognes, 1985: 371-382 (revision of Trypocalliphora); Shewell, in McAlpine et al., 1987: 1134-5, 1140, 1143; Sabrosky et al., 1989: 44.

\section{Protocalliphora $(P$.$) asiovora Shannon \& Dobroscky, 1924$}

Protocalliphora avium var. asiovora Shannon \& Dobroscky, 1924: 249. Type locality: USA, Washington, McElroy Lake.

Apaulina avium (Shannon \& Dobroscky): Hall, 1948: 186.

Apaulina basingeri Hall, 1948: 190. Type Locality: USA, California, San Diego.

Protocalliphora asiovora (Shannon \& Dobroscky): Hall, 1965: 926. 
Distribution: Canada, United States of America. Mexico (Baja California) (Sabrosky et al., 1989).

\section{Protocalliphora beameri Sabrosky, Bennett \& Whitworth, 1989}

Protocalliphora beameri Sabrosky, Bennett \& Whitworth, 1989: 113. Type locality: USA, Arizona, Cave Creek Canyon.

Protocalliphora n. sp. B of Horning \& Barr, 1970: 73.

Additional references: Sabrosky et al., 1989: 113.

Distribution: United States of America. Mexico (Baja California) (Sabrosky et al., 1989).

\section{Protocalliphora sialia Shannon \& Dobroscky, 1924}

Protocalliphora splendida var. sialia Shannon \& Dobroscky, 1924: 249. Lectotype locality: USA, Pennsylvania, Delaware River.

Protocalliphora chrysorrhoea (Meigen): Henshaw, 1908: 87. Type series: destroyed (Rognes, 1991).

Phormia chrysorrhoea (Meigen): Lloyd, 1922: 116.

Apaulina sialia (Shannon \& Dobroscky): Hall, 1948: 201.

Additional references: Sabrosky et al., 1989: 208 (lectotype designation).

Distribution: Alaska, Canada, United States of America. Mexico (Durango, Puebla) (Sabrosky et al., 1989).

Subfamily Luciliinae Shannon, 1923

Lucilini Shannon, 1923: 103, elevated to subfamily by Brues et al., 1954: 356.

\section{Genus Lucilia Robineau-Desvoidy, 1830}

Lucilia Robineau-Desvoidy, 1830: 452. Type species: Musca caesar Linnaeus, 1758. Subsequent designation of Macquart, 1834: 162.

Phaenicia Robineau-Desvoidy, 1863: 750. Type species: Phaenicia concinna Robineau-Desvoidy, 1863: 778 (Townsend, 1916b: 8). Some Authors in North America continued to use this as a subgenus or genus until Rognes (1991) and Stevens and Wall (1996) discussed their phylogenetic relationships.

Bufolucilia Townsend, 1919: 542. Type species: Lucilia bufonivora Moniez, 1876: 25.

Francilia Shannon, 1924: 74. Type species: Francilia alaskensis Shannon, 1924: 74.

Viridinsula Shannon, 1926: 131. Shannon used this name as a subgenus of Lucilia but Curran (1934) elevated the subgenus to genus, James (1966) degraded it again to subgenus, and posteriorly Rognes (1991) did not recognized any subgenera and listed Viridinsula as a synonym of Lucilia (See Whitworth, 2014).

\section{Lucilia cluvia (Walker, 1849)}

Musca cluvia Walker, 1849: 855. Type locality: West Indies.

Lucilia pilatei Hough, 1899a: 287. Type locality: USA, Georgia, Tifton. See Whitworth (2014) for further discussion on synonymy.

Lucilia cluvia (Walker): Shannon, 1926: 133.

Phaenicia cluvia (Walker): Hall, 1948: 236. 
Distribution: Widespread in North and Central America. Mexico (Chiapas, Veracruz, Oaxaca, Campeche, Nayarit) (Whitworth, 2014).

\section{Lucilia cuprina (Wiedemann, 1830)}

Musca cuprina Wiedemann, 1830: 654. Type locality: China.

Lucilia dorsalis Robineau-Desvoidy, 1830: 453. Type locality: Cape of Good Hope.

Lucilia amica Robineau-Desvoidy, 1830: 453. Type locality: Indonesia, Timor.

Lucilia elegans Robineau-Desvoidy, 1830: 458. Type locality: France, Ile de France.

Lucilia argyricephala Macquart, 1846: 326. Type locality: Cape of Good Hope (see Waterhouse \& Paramonov, 1950 as Lucilia argyrocephala Macquart).

Musca fucina Walker, 1849: 883. Type locality: South Africa.

Musca serenissima Walker, 1852: 340. Type locality: West Indies.

Musca temperata Walker, 1852: 340. Type locality: East Indies.

Lucilia leucodes Frauenfeld, 1867: 453. Type locality: "Im Chinesischen Meer".

Somomya pallifrons Bigot, 1877a: 257. Type locality: Australia.

Strongyloneura nigricornis Senior-White, 1924: 115. Type locality: India, North West Frontier Post, Cherat.

Lucilia pallescens Shannon, 1924: 78. Type locality: USA, North Carolina, Wilmington.

Lucilia cuprina (Wiedemann): Shannon, 1926: 131.

Additional references: Waterhouse \& Paramonov, 1950.

Distribution. This species has an almost worldwide distribution; in America it has stablished from southern United States through Central America to Northern Argentina and Peru. Mexico (Veracruz) (Whitworth, 2014).

\section{Lucilia eximia (Wiedemann, 1819)}

Musca eximia Wiedemann, 1819: 53. Type locality: Brazil.

Lucilia smaragdula Robineau-Desvoidy, 1830: 462. Type locality: Brazil.

Lucilia punctipennis Macquart, 1848: 216. Type locality: Brazil.

Lucilia subrectineuris Macquart, 1851: 223. Type locality: Brazil, Minas Gerais.

Musca insularis Walker, 1853: 340. Type locality: West Indies.

Somomya amazona Bigot, 1877a: 255. Type locality: Brazil.

Somomyia mutabilis Bigot, 1877a: 248. Type locality: "Mexicus".

Somomyia pueblensis Bigot, 1877a: 250. Type locality: "Mexicus".

Somoyia sylphida Bigot, 1877b: 45. Type locality: USA, New Orleans.

Lucilia hirtiforceps Shannon, 1926: 133. Type locality: Panama, Canal Zone.

Lucilia mera Shannon \& Del Ponte, 1926: 586. Type locality: Argentina, San Pedro de Jujuy.

Lucilia primaveris Shannon \& Del Ponte, 1926: 586. Type locality: Argentina, Buenos Aires, San Isidro.

Lucilia eximia (Wiedemann): Aubertin, 1933: 423.

Lucilia littoralis Blanchard, 1938: 380. Type locality: Argentina (see Whitworth, 2014).

Phaenicia eximia (Wiedemann): Hall, 1948: 239.

Additional references: Hall, 1948: 239; James, 1970: 10; Whitworth, 2014: 26.

Distribution: Widespread from southern United States through Central and South America. Mexico (Campeche, Chiapas, Guerrero, Jalisco, Estado de México, Michoacán, Morelos, Nuevo León, Oaxaca, Quintana Roo, San Luis Potosí, Sinaloa, Sonora, Tamaulipas, Veracruz) (Whitworth, 2014). 
Musca illustris Meigen, 1826: 54. Type locality: Germany. Musca parvula Meigen, 1826: 55. Type locality: Europe. Musca equestris Meigen, 1826: 57. Type locality: Europe. Lucilia fraterna Macquart, 1848: 217. Type locality: "America septentrionale". Lucilia consobrina Macquart, 1848: 217. Type locality: "America septentrionale". Musca muralis Walker, 1849: 888. Type locality: Canada, Hudson's Bay, Albany River. Calliphora simulatrix Pandelle, 1896: 218. Type locality: France.

Lucilia purpurea Townsend, 1908: 122. Type locality: USA, Alaska, Fort Wrangel (see Hall, 1948). Lucilia ilustris (Meigen): Collin, 1926: 258.

Distribution: Holarctic, from Canada to Northern Mexico (Hall, 1948; James, 1970; Whitworth, 2014).

\section{Lucilia mexicana Macquart, 1843}

Lucilia mexicana Macquart, 1843: 300. Type locality: Mexico.

Lucilia unicolor Townsend, 1908: 121. Type locality: USA, New Mexico. Lucilia infuscata Townsend, 1908: 123. Type locality: USA, New Mexico. Phaenicia mexicana (Macquart): Hall, 1948: 243.

Distribution: Southwestern United States of America to Honduras. Mexico (Baja California, Chiapas, Chihuahua, Coahuila, Durango, Jalisco, Michoacán, Morelos, Nuevo León, Oaxaca, Puebla, Zacatecas) (Whitworth, 2014).

\section{Lucilia purpurascens (Walker, 1836)}

Musca purpurascens Walker, 1836: 355. Type locality: Brazil, Santa Catarina.

Lucilia brunnicornis Macquart, 1843: 299. Type locality: "Mexique".

Lucilia violacea Macquart, 1847: 99. Type locality: "Mexique".

Lucilia ocularis Shannon, 1926: 132. Type locality: Costa Rica, San Mateo. There is a misspelling as "Lucilia oculatis" (see Whitworth (2014) for explanation).

Lucilia purpurascens (Walker): Aubertin, 1933: 426.

Phaenicia purpurescens (Walker): Hall, 1948: 254. Misspelling and subsequent authors mistake (see Whitworth, 2014).

Distribution: Mainly Neotropical from Mexico to northern Argentina. Mexico (Chiapas, Veracruz, Morelos, Tamaulipas, Ciudad de México) (Whitworth, 2014).

\section{Lucilia sericata (Meigen, 1826)}

Musca sericata Meigen, 1826: 53. Type locality: Germany.

Musca nobilis Meigen, 1826: 56. Type locality: Europe.

Chrsomya capensis Robineau-Desvoidy, 1830: 451. Type locality: Africa.

Lucilia basalis Macquart, 1842: 305. Type locality: Not specified.

Lucilia flavidipennis Macquart, 1843: 296. Type locality: Ceylon (Sri Lanka).

Musca lagyra Walker, 1849: 885. Type locality: Fayal.

Lucilia latifrons Schiner, 1862: 590. Type locality: Not specified.

Lucilia sayi Jaennicke, 1867: 375. Type locality: USA, Illinois.

Lucilia giralti Townsend, 1908: 121. Type locality: France, Paris.

Lucilia barberi Townsend, 1908: 121. Type locality: USA, Arizona, Williams. 
Lucilia frontalis Brauer \& Bergenstamm, 1891: 116 (nomen nudum). Aubertin, 1933: 411. Type locality: Egypt.

Lucilia sericata (Meigen): Townsend, 1908: 120.

Additional references: Mello, 1961: 261; James, 1970 for synonymy and discussion, and Whitworth (2012), for description and distribution.

Distribution: From southern Canada to Argentina. It is commonly stated that the species has a widespread distribution in the Americas, but it is doubtful (Hall, 1948; Mello, 1961; Whitworth, 2014). Mexico (Coahuila, San Luis Potosí) (Valdés-Perezgasga \& García-Espinoza, 2014).

\section{Lucilia silvarum (Meigen, 1826)}

Musca silvarum Meigen, 1826: 53. Type locality: Not specified.

Lucilia brunicosa Robineau-Desvoidy, 1830: 459. Type locality: North America.

Lucilia nigripalpis Townsend, 1908: 120. Type locality: USA, Ohio.

Bufolucilia lucilioides Wulp, 1896: 288. Enderlein, 1933: 120 (as Xerophilophaga). Type locality: Mexico, Mexico City.

Bufolucilia silvarum (Meigen): Townsend, 1919: 542; Hall, 1948: 219.

Lucilia silvarum (Meigen): Rognes, 1991: 181.

Additional references: Hall, 1948: 219; Tantawi \& Whitworth, 2014: 104 (Key).

Distribution: Holarctic: Europe, Asia, North Africa (Rognes, 1991); Nearctic: Canada, United States of America. Mexico (Ciudad de México, Coahuila) (Hall, 1948, 1965; James, 1970; Valdés-Perezgasga \& García-Espinoza, 2014).

\section{Subfamily Chrysomyinae Shannon, 1923}

Chrysomyini Shannon, 1923: 103, elevated to subfamily by Malloch, 1927: 299, 300, 324.

\section{Genus Cochliomyia Townsend, 1915}

Callitroga Brauer, 1883: 74. Type species: Musca macellaria Fabricius, 1775: 776. Type locality: West Indies (see Melville, 1984, name suppression).

Cochliomyia Townsend, 1915c: 646. Type species: Musca macellaria Fabricius.

\section{Cochliomyia hominivorax (Coquerel, 1858)}

Lucilia hominivorax Coquerel, 1858: 173. Type locality: French Guiana, Cayenne.

Compsomyia homicida Brauer, 1899: 525, misspelling of hominivorax Coquerel.

Calliphora infesta Philippi, 1861: 513. Type locality: Chile, Santiago.

Calliphora anthropophaga Conil, 1878: 71. Type locality: Argentina, Córdoba.

Somomyia fulvobarbata Bigot, 1888: 598. Type locality: Uruguay, Montevideo.

Cochliomyia americana Cushing \& Patton, 1933: 539. Type locality: USA, Texas, Menard.

Cochliomyia hominivorax (Coquerel): Dear, 1985: 138.

Additional references: Dear, 1985: 138. (taxonomy discussion). 
Distribution: Argentina, Brazil, Chile, Colombia, Costa Rica, Cuba, Dominican Republic, French Guyana, Greater Antilles, Guatemala, Jamaica, Nicaragua, Panama, Peru, Puerto Rico, Trinidad and Tobago, Uruguay. Mexico (Veracruz, Tamaulipas, Yucatán, Chiapas) (Dear, 1985; Wolff \& Kossman, 2016).

\section{Cochliomyia macellaria (Fabricius, 1775)}

Musca macellaria Fabricius, 1775: 776. Type locality: "America".

Musca laniaria Wiedemann, 1830: 683. Type locality: Brazil.

Chrysomya viridula Robineau-Desvoidy, 1830: 445. Type locality: Brazil.

Chrysomya affinis Robineau-Desvoidy, 1830: 445. Type locality: Brazil.

Chrysomya tibialis Robineau-Desvoidy, 1830: 446. Type locality: Jamaica, Pour-Au-Prince.

Chrysomya lherminieri Robineau-Desvoidy, 1830: 446. Type locality: USA, Carolina.

Chrysomya alia Robineau-Desvoidy, 1830: 447. Type locality: Brazil.

Chrysomya socia Robineau-Desvoidy, 1830: 447. Type locality: Brazil.

Chrysomya decora Robineau-Desvoidy, 1830: 448. Type locality: "Antilles".

Chrysomya plaei Robineau-Desvoidy, 1830: 448. Type locality: "Antilles".

Chrysomya lepida Robineau-Desvoidy, 1830: 448. Type locality: Brazil.

Calliphora violacea Macquart, 1843: 285. Type locality: Africa, probably a mistake. Dear (1985) states that it is from Chile.

Lucilia vittata Macquart, 1843: 298. Type locality: Australia, New-Holland. Dear (1985) mentioned a locality mistake, because it is a species from the New World.

Lucilia durvillei Macquart, 1843: 299. Type locality: Peru, Payta.

Musca certima Walker, 1849: 873. Type locality: USA, Florida.

Musca phauda Walker, 1849: 896. Type locality: Galapagos Islands.

Pyrellia cyanicolor Rondani, 1850: 178, unjustified name for Calliphora violacea Macquart.

Calliphora tristriata Verhuell, 1850: 273. Type locality: Surinam, Paramaribo, Paramaribo.

Calliphora tibialis Macquart, 1851: 215. Type locality: Brazil, Rio de Janeiro.

Lucilia rubrifrons Macquart, 1851: 223. Type locality: Argentina, Buenos Aires.

Musca turbida Walker, 1853: 336. Type locality: Jamaica.

Musca fasciata Walker, 1853: 337. Type locality: Brazil.

Lucilia picicrus Thomson, 1869: 543. Type locality: Panama.

Lucilia curvipes Thomson, 1869: 544. Type locality: Brazil, Río de Janeiro.

Lucilia porticola Thomson, 1869: 544. Type locality: Peru, Callao.

Lucilia quadrisignata Thomson, 1869: 544. Type locality: Galapagos Islands.

Somomya flavigena Bigot, 1877a: 249. Type locality: Mexico.

Somomya aztequina Bigot, 1877a: 252. Type locality: Mexico.

Somomyia iridicolor Bigot, 1888: 599. Type locality: Cuba.

Chrysomyia lynchi Lahille, 1915: 12. Type locality: Argentina, Chaco.

Cochliomyia fontanai García, 1952: 71. Type locality: Argentina, Belgrano.

Distribution: This species is common in the Nearctic and Neotropical regions. Argentina, Bahamas, Belize, Bermuda, Bolivia, Brazil, Caribbean, Chile, Costa Rica, Cuba, Dominican Republic, Ecuador, Greater Antilles, Guatemala, Guyana, Honduras, Jamaica, Nicaragua, Panama, Paraguay, Peru, Trinidad and Tobago, Uruguay, Venezuela. Mexico (Veracruz, Jalisco, Yucatán, Chiapas, Morelos, Durango) (Dear, 1985; Whitworth, 2010; Wolff \& Kosmann, 2016).

\section{Genus Compsomyiops Townsend, 1918}

Compsomyiops Townsend, 1918: 153. Type species: Calliphora fulvipes Macquart, 1843: 289.

Myiolucilia Hall, 1948: 109. Type species: Musca lyrcea Walker, 1849: 873. 
Compsomyiops callipes (Bigot, 1877)

Somomya callipes Bigot, 1877a: 249. Type locality: Mexico.

Somomya calopus Bertkau, 1879: 538 (unjustified emendation of callipes).

Chrysomyia wheeleri Hough, 1899a: 284. Type locality: USA, California, Monterey.

Paralucilia wheeleri (Hough): Hall, 1948: 153.

Distribution: United States of America. Mexico (Ciudad de México, Guerrero, Michoacán, Morelos, Veracruz) (Dear, 1985).

\section{Compsomyiops fulvicrura (Robineau-Desvoidy, 1830)}

Chrysomya fulvicrura Robineau-Desvoidy, 1830: 446. Type locality: Uruguay, Montevideo.

Calliphora fulvipes Macquart, 1843: 289. Type locality: Chile.

Musca lyrcea Walker, 1849: 873. Type locality: Montevideo, Uruguay.

Musca caruca Walker, 1849: 877. Type locality: Chile.

Musca gamelia Walker, 1849: 878. Type locality: Uruguay, Montevideo.

Calliphora annulipes Philippi, 1861: 514. Type locality: Chile, Santiago.

Lucilia taeniaria Thomson, 1869: 544. Type locality: Argentina, Buenos Aires.

Paralucilia nigripes Mello, 1968: 184. Type locality: Argentina, Buenos Aires, Boulogne.

Distribution: Argentina, Brazil, Chile, Guyana, Mexico, Uruguay (Lopes \& Albuquerque, 1955; James, 1970; Guimarães et al., 1983; Kosmann et al., 2013). However, there is not a punctual record for Mexico, as stated above many authors use the distribution of this species as it is spread through Mexico, Central and South America. But according to Dear (1985), González-Mora et al. (1998) and Mariluis and Mueleri (2003), the distribution is restricted to South America (Bolivia, Guyana, Brazil, Uruguay, Argentina, Chile). Dear (1985) stated that there has been a lot of misidentifications thorough history, and confusion specially with Hall (1948) (See the discussion under Paralucilia in Dear, 1985), so this species is recorded for Mexico in many works, but it may or may not be present in Mexico as we could not find a punctual record, further analysis and material revision is required to confirm this.

\section{Compsomyiops melloi Dear, 1985}

Compsomyiops melloi Dear, 1985: 155. Type locality: Mexico, Ciudad (de México).

Distribution: Colombia. Mexico (Ciudad de México) (Dear, 1985; Kosmann et al., 2013).

Genus Chloroprocta Wulp, 1896

Chloroprocta Wulp, 1896: 296. Type species: Chloroprocta semiviridis Wulp, 1896: 296 (= Chrysomya idiodea Robineau-Desvoidy, 1830).

Callitrogopsis Townsend, 1935: 70. Type species: Callitrogopsis costalis Townsend, 1935: 70 (= Chrysomya idiodea Robineau-Desvoidy, 1830).

Chloroprocta idiodea (Robineau-Desvoidy, 1830)

Chrysomya idiodea Robineau-Desvoidy, 1830: 445. Type locality: Brazil.

Musca violacea Fabricius, 1805: 228. Type locality: "America Meridionalis".

Lucilia fuscanipennis Macquart, 1851: 223. Type locality: Brazil.

Musca purpurea Walker, 1853: 337. Type locality: South America.

Chloroprocta semiviridis Wulp, 1896: 296. Type locality: Mexico, Yucatán (Type designation of Townsend, 1937: 123). 
Strongyloneura flavifacies Engel, 1931: 138. Type locality: Bolivia.

Callitrogopsis costalis Townsend, 1935: 71. Type locality: Brazil (type designation of Townsend, 1937: 123).

Additional references: James, 1970: 5; Dear, 1985: 113; Pape et al., 2004: 203; Amat, 2009: 700; Amat, 2010: 399; Kosmann et al., 2013: 76; Wolff \& Kosmann, 2016: 859.

Distribution: Bahamas, Brazil, Colombia, Cuba, El Salvador, Guyana, Nicaragua, Panama, Paraguay, Peru, Trinidad, United States of America, Venezuela (Kosmann et al., 2013). México (Colima, Yucatán) (Dear, 1985; Kosmann et al., 2013).

\section{Genus Chrysomya Robineau-Desvoidy, 1830}

Chrysomya Robineau-Desvoidy, 1830: 444. Type species: Chrysomya regalis Robineau-Desvoidy, 1830: 444, by designation of Coquillett, 1910: 523 .

Compsomyia Rondani, 1875: 425. Type Species: Musca dux Eschscholtz.

Pycnosoma Brauer \& Bergenstamm, 1894: 623. Type species: Musca marginalis Wiedemann.

Paracompsomyia Hough, 1898c: 184. Type species: Paracompsomyia nigripennis Hough.

Psilostoma Surcouf, 1919: 58. Type species: Ochromyia incisuralis Macquart.

Microcalliphora Townsend, 1916a, 618. Type species: Lucilia varipes Macquart.

Cyaneosomyia Séguy, 1928: 112. Type species: Cyaneosomyia phaonis Seguy.

Pycnosomops Townsend, 1934b: 277. Type species: Musca putoria Wiedemann.

Additional references: Dear, 1985: 112; Zumpt, 1956: 178 (taxonomic review of the genus).

Chrysomya megacephala (Fabricius, 1794)

Musca megacephala Fabricius, 1794: 317. Type locality: India, Teshi, Gahna.

Musca dux Eschscholtz, 1822: 114. Type locality: Guam.

Chrysomya duvaucelii Robineau-Desvoidy, 1830: 451. Type locality: India, West Bengai.

Chrysomya gratiosa Robineau-Desvoidy, 1830: 451. Type locality: "Oriental Region”.

Lucilia flaviceps Macquart, 1843: 302. Type locality: India.

Musca remuria Walker, 1849: 871. Type locality: China.

Musca bata Walker, 1849: 875. Type locality: Not specified.

Musca combrea Walker, 1849: 876. Type locality: Not specified.

Pollenia basalis Smith, 1876: 449. Type locality: Indic ocean, Isla Rodrigues.

Somomyia pfefferi Bigot, 1877a: 257. Type locality: Mauritius.

Somomyia cyanocincta Bigot, 1888: 604. Type locality: Indonesia, Java.

Somomyia dives Bigot, 1888: 600. Type locality: India, Calcutta.

Chrysomya megacephala (Facribius): Malloch, 1930: 233.

Additional references: Senior-White et al., 1940: 138 (taxonomic review and keys); Kurahashi, 1991: 713; Zumpt, 1965: 97.

Distribution: From North to South America (Dear, 1985). Mexico (Baja California Sur, Chiapas, Estado de México, Michoacán, Morelos, Veracruz, Yucatán) (Castañeda-Vildózola et al., 1999; Pérez-Balam et al., 2012).

Chrysomya rufifacies (Macquart, 1843)

Lucilia rufifacies Macquart, 1843: 303. Type locality: Australia, New Holland.

Lucilia orientalis Macquart, 1843: 302. Type locality: India, Pondicherry. 
Lucilia pavonina Schiner, 1868: 305. Type locality: Kar Nicobar and Tellschong (Andaman and Nicobar Islands).

Somomyia barbata Bigot, 1877b: 30. Type locality: India.

Chrysomya cordieri Séguy, 1925: 303. Type locality: Indonesia, Java, Sockaboemi.

Chrysomyia rufifacies (Macquart) Bezzi, 1927: 235.

Additional references: Holdaway, 1933: 553; Senior-White et al., 1940: 141; Zumpt, 1965: 92 (taxonomic review and history); Kurahashi, 1991: 713.

Distribution: Stablished in almost all the Americas (Dear, 1985). Mexico (Chiapas to Durango) (Gagné, 1981; Baumgartner \& Greenberg, 1984).

\section{Genus Hemilucilia Brauer, 1895}

Hemilucilia Brauer, 1895: 598. Type species: Musca segmentaria Fabricius, 1805: 292.

Mya Rondani, 1850: 175, primary homonym.

Additional references: Dear, 1985: 124 (species review).

\section{Hemilucilia segmentaria (Fabricius, 1805)}

Musca segmentaria Fabricius, 1805: 292. Type locality "America Meridionalis". Additional references: Dear (1985); Kosmann et al. (2013) (taxonomic review and keys).

Lucilia nubipennis Rondani, 1848: 77. New name for "segmentaria" of Wiedemann.

Chrysomya hyacinthina Robineau-Desvoidy, 1830: 450. Type locality: South America.

Calliphora femorata Walker, 1861: 310. Type locality: Mexico.

Hemilucilia hermanlenti Mello, 1972b: 548. Type locality: Brazil, Río de Janeiro.

Hemilucilia nubipennis occidentalis Mariluis, 1980: 86. Type locality: Ecuador.

Hemilucilia segmentaria (Fabricius): Dear, 1985: 127.

Distribution: Argentina, Bolivia, Brazil, Colombia, Costa Rica, Ecuador, El Salvador, Guyana, Mexico, Panama, Paraguay, Peru, Trinidad (Dear, 1985). Mexico (Morelos, Veracruz, personal observations).

\section{Hemilucilia semidiaphana (Rondani, 1850)}

Mya semidiaphana Rondani, 1850: 177. Type locality: Brazil.

Hemilucilia hermanlenti Mello, 1972b: 548. Type locality: Brazil, Río de Janeiro.

Hemilucilia segmentaria ssp. pacifiencis Mariluis, 1980: 85. Type locality: Ecuador.

Hemilucilia semidiaphana (Rondani): Dear, 1985: 130.

Additional references: Dear, 1985: 130 (discussion of types and taxonomic review).

Distribution: From Argentina to Mexico (Dear, 1985). Mexico (Veracruz, personal observation).

\section{Genus Paralucilia Brauer \& Bergenstamm, 1891}

Paralucilia Brauer \& Bergenstamm, 1891: 391. Type species: Calliphora fulvipes Macquart, 1834: 289.

Additional references: Dear, 1985: 117 (review of species; commentaries on type species specimen). 
Paralucilia fulvinota (Bigot, 1877)

Somomya fulvinota Bigot, 1877a: 251. Type locality: Mexico.

Chrysomyia desvoidy Hough, 1900: 208. Type locality: Brazil, Chapada.

Paralucilia braueri Townsend, 1916b: 11. Type locality: Chile.

Paralucilia fulvinota (Bigot): Dear, 1985: 119.

Additional references: Dear, 1985: 119 (discussion about types and taxonomy).

Distribution: Bolivia, Brazil, Chile, Costa Rica, Colombia, Ecuador, Guyana, Peru, Venezuela. Mexico (Morelos, Veracruz) (Dear, 1985).

\section{Genus Phormia Robineau-Desvoidy, 1830}

Phormia Robineau-Desvoidy, 1830: 465. Type species: Musca regina Meigen, 1826: 58.

Euphormia Townsend, 1919: 542. Type species: Musca regina Meigen, 1826: 58.

\section{Phormia regina (Meigen, 1826)}

Musca regina Meigen, 1826: 58. Type locality: Not specified.

Musca thalassina Meigen, 1826: 54. Type locality: Europe.

Phormia philadelphica Robineau-Desvoidy, 1830: 466. Type locality: USA, Pennsylvania.

Phormia regina (Meigen): Robineau-Desvoidy, 1830: 466.

Phormia cuprea Robineau-Desvoidy, 1830: 467. Type locality: France, Paris.

Phormia fulvifacies Robineau-Desvoidy, 1830: 467. Type locality: France, Paris.

Phormia vittata Robineau-Desvoidy, 1830: 467. Type locality: France, Yonne.

Phormia squalens Robineau-Desvoidy, 1830: 468. Type locality: France, Yonne.

Musca mollis Walker, 1849: 892. Type locality: Canada, Ontario.

Musca proxima Walker, 1853: 341: Type locality: USA, California.

Lucilia rufipalpis Jaennicke, 1867: 375. Type locality: USA, Illinois.

Lucilia stigmaticalis Thomson, 1869: 544. Type locality: USA, California.

Somomya nigrina Bigot, 1877a: 247. Type locality: USA, Illinois.

Somomyia rupicola Bigot, 1888: clxxx (180). Type locality: USA, Rocky Mountains.

Somomyia rufigena Bigot, 1888: clxxxi (181). Type locality: USA, Rocky Mountains.

Distribution: Holarctic: Canada, United States of America. In Mexico as south as Mexico City (Hall, 1948), but also in Veracruz (personal observation).

ACKNOWLEDGEMENTS. The first author was supported by a scholarship from the Mexican Council of Science and Technology (CONACYT, No. 634637) as part of the work "Diversidad de moscas carroñeras de la familia Calliphoridae (Diptera) en tres sitios con diferente grado de antropización en la ciudad de Xalapa, Veracruz, México". It was also supported by the project "Actualización del Catálogo de Autoridad Taxonómica del Orden Diptera (Insecta) de México" (CONABIO JE006) and (INECOL-10816), granted to Sergio Ibáñez-Bernal.

\section{LITERATURE CITED}

Aldrich, J. M. (1901) Synonymische Notiz. Wiener Entomologische Zeitung, 20, 68.

Aldrich, J. M. (1905) A catalogue of North American Diptera. Smithsonian Miscellaneous Collections, 46, $680 \mathrm{pp}$. 
https://doi.org/10.5962/bhl.title.1681

Aldrich, J. M. (1930) New two-winged flies of the family Calliphoridae from China. Proceedings of the United States Natural Museum, 78, 1-5. https://doi.org/10.5479/si.00963801.78-2844.1

Amat, E. (2009) Contribución al conocimiento de las Chrysomyinae y Toxotarsinae (Diptera: Calliphoridae) de Colombia. Revista Mexicana de Biodiversidad, 80, 693-708.

Amat, E. (2010) Notes on necrophagous flies (Diptera: Calyptratae) associated to fish carrion in Colombian Amazon. Acta Amazonica, 40, 397-400. https://doi.org/10.1590/s0044-59672010000200018

Aubertin, D. (1933) Revision of the genus Lucilia R.-D. (Diptera, Calliphoridae). Journal of the Linnaean Society of London, Zoology, 38, 389-436. https://doi.org/10.1111/j.1096-3642.1933.tb00991.x

Baumgartner, D. L., Greenberg, B. (1984) The genus Chrysomya (Diptera: Calliphoridae) in the New World. Journal of Medical Entomology, 1, 105-113. https://doi.org/10.1093/jmedent/21.1.105

Bertkau, P. (1879) Diptera. Bericht über die wissenschaftlichen Leistungen im Gebiete der Entomologie, $1877-1878,518-542$.

Bezzi, M. (1927) Some Calliphoridae (Diptera) from the South Pacific islands and Australia. Bulletin of Entomological Research, 17, 231-247. https://doi.org/10.1017/S000748530001928

Bigot, J. M. F. (1877a) Dipteres nouveaux ou peu connus. 7 partie, IX: Genre Somomyia (Rondani) Lucilia (Rob.-Desv.) Calliphora, Phormia, Chrysomyia (id). Robineau-Desvoidy. Annales de la Société entomologique de France, ser. 5, 35-48. https://doi.org/10.5962/bhl.title.9278

Bigot, J. M. F. (1877b) Dipteres nouveaux ou peu connus. 8 partie, X: Genre Somomya (Rondani) Calliphora, Melinda, Mufetia, Lucilia, Chrysomyia (alias Microchrysa Rond.) Robineau-Desvoidy. Annales de la Société entomologique de France, ser. 5, 243-259. https://doi.org/10.5962/bhl.title.9278

Bigot, J. M. F. (1888) Dipteres nouveaux ou peu connus. Muscidi (J.B.) Bulletin de la Société zoologique de France, 12, 581-617.

Blanchard, E. E. (1938) Descripciones y anotaciones de dipteros argentinos. Anales de la Sociedad Científica Argentina, 126, 345-386.

Brauer, F. (1883) Die Zweiflugler des Kaiserlichen Museums zu Wien. III. Denkschriften der Kaiserlichen Akademie der Wissenschaften. Wien. Mathematisch-Naturwissenschaftliche Classe, 47, 1-100.

Brauer, F. (1893) Vorarbeiten zu einer Monographie der Muscaria schizometopa (exclusive Anthomyidae) von Prof. Dr. Fr. Bauer und Julius Edl. v. Bergenstamm. Verhandlungen des ZoologischBotanischen Vereins in Wien, 43, 447-525.

Brauer, F. (1895) Bemerkungen zu einigen neuen Gattungen der Muscarien und Deutung einiger OriginalExemplare. Sitzungsberichte, 104, 582-604.

Brauer, F. (1899) Beitrage zur Kenntniss der Muscaria Schizometopa. I. Bemerkungen zu den Originalexemplaren der von Bigot, Macquart und Robineau-Desvoidy beschriebenen Muscaria Schizometopa aus der Sammlung des Herrn G.H. Verrall. Denkschriften der Kaiserlichen Akademie der Wissenschaften. Wien. Mathematisch-Naturwissenschaftliche Classe, 108, 495-529.

Brauer, F., Bergenstamm, J. E. von (1889) Die Zweiflugler des Kaiserlichen Museums zu Wien. IV. Vorarbeiten zu einer Monographie der Muscaria Schizometopa (exclusive Anthomyidae). Pars I. Denkschriften der Kaiserlichen Akademie der Wissenschaften Wien. MathematischNaturwissenschaftliche Classe, 56, 69-180. https://doi.org/10.1002/mmnd.18900330231

Brauer, F., Bergenstamm, J. E. von. (1891) Die Zweiflügler des Kaiserlichen Museums zu Wien. V. Vorarbeiten zu einer Monographie der Muscaria Schizometopa (exclusive Anthomyidae). Pars II. 
Denkschriften der Kaiserlichen Akademie der Wissenschaften. Wien. MathematischNaturwissenschaftliche Classe, 58, 305-446.

Brauer, F., Bergenstamm, J. E. von. (1894) Die Zweiflügler des Kaiserlichen Museums zu Wien. VII. Vorarbeiten zu einer Monographie der Muscaria Schizometopa (exclusive Anthomyidae). Pars IV, Denkschriften der Kaiserlichen Akademie der Wissenschaften. Wien. MathematischNaturwissenschaftliche Classe, 61, 537-624.

Brues, C. T., Melander, A. L., Carpenter, F. M. (1954) Classification of insects. (Revised ed.). Bulletin of the Museum of Comparative Zoology at Harvard College, 108, 917 pp.

Byrd, H. J., Castner, L. J. (2010) Forensic Entomology: The utility of Arthropods in Legal Investigations. CRC Press, United States of America, 708 pp.

Castañeda-Vildózola, A., Equihua-Martínez, A., Valdés-Carrasco, J., Barrientos-Priego, A. F., IshAm, G., Gazit, S. (1999) Insectos Polinizadores del Aguacatero en los estados de México y Michoacán, Revista Chapingo Serie Horticultura, 5, 129-136.

Cerretti, P., Stireman, J. O. III, Pape, T., O'Hara, J. E., Marinho, M. A. T., Rognes, K. (2017) First fossil of an oestroid fly (Diptera: Calyptratae: Oestroidea) and the dating of oestroid divergences. PLOS ONE, 12, e0182101. https://doi.org/10.1371/journal.pone.0182101

Collin, J. E. (1926) Supplementary Notes on the Genus Lucilia, Desv. (Diptera). Transactions of the Royal Entomological Society of London, 74, 258-260. https://doi.org/10.1111/j.1365-2311.1926.tb02234.x

Conil, P. A. (1878) Myiasis, pp. 43-98. In: C. Lesbini, C. H. Weyenbergh, P. A. Conil (Eds.). Etudes sur la Myasis. Actas de la Academia Nacional de Ciencias de la República Argentina en Córdoba, 3. https://doi.org/10.5962/bhl.title.9347

Coquerel, C. (1858) Note sur les larvae appartenant à une espèce nouvelle de Diptère (Lucilia hominivorax) developpèes dans les frontaux de l'homme à Cayenne. Annales de la Société Entomologique de France, 3, 171-176.

Coquillett, D. W. (1910) The type-species of the North American genera of Diptera. Proceedings of the United States National Museum, 37, 499-647.

Curran, C. H. (1934) The Templeton Crocker Expedition of the California Academy of Sciences. Proceedings of the California Academy of Sciences, 21, 147-172.

Cushing, E. C., Patton, W. S. (1933) Studies on the higher Diptera of medical and veterinary importance. Cochliomyia americana sp. nov., the screw-worm fly of the New World. Annals of Tropical Medicine and Parasitology, 27, 539-551. https://doi.org/10.1080/00034983.1933.11684779

Dear, J. P. (1985) A revision of the new world Chrysomyini (Diptera: Calliphoridae). Revista Brasileira de Zoologia, 3, 109-169. http://dx.doi.org/10.1590/S0101-81751985000300001

De Geer, C. (1776) Mémoires pour servir à l'histoire des Insectes. Vol. 6. Hesselberg, Stockholm, viii + $523 \mathrm{pp}$.

Enderlein, G. (1933) Neue paläarktischen Calliphoriden darunter Schneckenparasiten (Dipt.). Mitteilungen der Deutschen Entomologischen Gesellschaft, 4, 120-128.

Engel, O. (1931) Die Ausbeute der deutschen Chaco-Expedition 1925/26. Diptera. (Fortsetzung.) XXVI. Anthomyidae, XXVII. Muscidae und XXVIII. Sarcophagidae. Konowia, 10, 133-154.

Eschscholtz, J. F. (1822) Entomographien. Erste Lieferung. Reimer, Berlin, 128 pp.

Fabricius, J. C. (1775) Systema entomologiae, sistens insectorum classes, ordines, genera, species, adiectis synonymis, locis, descriptionibus, observationibus. Kortii, Flensbvrgi et Lipsiae (= Flensburg and Leipzig), $832 \mathrm{pp}$. https://doi.org/10.5962/bhl.title.36510

Fabricius, J. C. (1794) Entomologia systematica emendata et aucta. Secundum classes, ordines, genera, species adjectis synonimis, locis, observationibus, descriptionibus. Tom. IV. C. G. Proft, Fil. et Soc., Hafniae [= Copenhagen], 472 pp. 
Fabricius, J. C. (1805) Systema antliatorum secundum ordines, genera, species adiectis synonymis, locis, observationibus, descriptionibus. Apun Carolum Reichard, Brunsvigae, 372 pp.

Fallén, C. F. (1817) Beskrifning ofver de i Sverige funna Fluge Arter, som kunna foras till slagtet Musca. Forsta Afdelningen. Kongliga Vetenskaps- Academiens Handlingar, 226-254.

Flores, P. L. R. (2009) Sucesión de entomofauna cadavérica utilizando como biomodelo cerdo blanco ( $S u s$ scrofa L.). Tesis de Doctorado. Colegio de Postgraduados. Estado de México.

Frauenfeld, G. R. von. (1867) Zoologische miscellen XI. Verhandlungen der Zoologisch-Botanischen Gesellschaft in Wien, 17, 425-502.

Gagné, R. J. (1981) Chrysomya Spp., Old World blow flies (Diptera: Calliphoridae), recently established in the Americas. Bulletin of the Entomological Society of America, 27, 21-22. https://doi.org/10.1093/besa/27.1.21

García, M. (1952) Consideraciones generales sobre el género Cochliomyia Townsend, 1916 y descripción de C. fontanai n. sp. (Diptera Calliphoridae). Publicaciones del Instituto Regional de Entomología Sanitaria, 1-8, 68-80.

García-Espinoza, F., Valdés-Perezgasga, Ma. T., Sánchez-Ramos, F. J., Yussef-Vanegas, S. Z., Quintero-Martínez, Ma. T. (2012) Desarrollo larval y requerimientos calóricos de Chrysomya rufifacies (Diptera: Calliphoridae) durante primavera y verano en Torreón Coahuila. Acta Zoológica Mexicana (nueva serie), 28, 172-184. https://doi.org/10.21829/azm.2012.281824

González-Mora, D., Peris, S. V., Mariluis, J. C. (1998) Notas sobre la taxonomía y distribución del género Compsomyiops Townsend, 1908 (Diptera, Calliphoridae). Boletín de la Real Sociedad Española de Historia Natural (Sección Biología), 94, 15-21.

Guimarães, J. H., Papavero, N., Prado, A. P. do (1983) As miíases na região neotropical (Identificação, biología, bibliografia). Revista brasileira Zoologia, 1, 239-416. https://doi.org/10.1590/s0101-81751982000400001

Hamerstrom, F., Hamerstrom, F. (1954) Myiasis of the ears of hawks. Falconry News and Notes, 1, 4-8.

Halffter, G. (2003) Biogeografía de la entomofauna de montaña de México y América Central, pp. 87-97. In: J. J. Morrone, J. Llorente (Eds.). Una perspectiva latinoamericana de la biogeografía. Las Prensas de Ciencias, Facultad de Ciencias, Universidad Nacional Autónoma de México, México D.F.

Hall, D. G. (1948) The blowflies of North America. Lafayette, Indiana, Thomas Say Foundation, 477 pp.

Hall, D. G. (1965) Calliphoridae, pp. 922-933. In: A. Stone, C. W. Sabrosky, W. W. Wirth, R- H. Foote, J. R. Coulson. (Eds.). A catalog of the Diptera of America north of Mexico. Agricultural Research Service United States Department of Agriculture, $1696 \mathrm{pp}$.

Hendel, F. (1901) Beitrag zur Kenntnis der Calliphorinen (Dipt.). Wiener Entomologische Zeitung, 20, 2833.

Hendel, F. (1911) Uber von Professor J. M. Aldrich erhaltene und einige andere amerikanische Dipteren. Wiener entomologische Zeitung, 30, 19-46.

Henshaw, H. W. (1908) A parasitic fly injurious to our native birds. Auk, 25, 87-88.

Holdaway, F. G. (1933) The synonymy and Distribution of Chrysomya rufifacies (Macq.), and Australian sheep blowfly. Bulletin of Entomological Research, 24, 549-560. https://doi.org/10.1017/S0007485300035501

Horning, D. S., Barr, W. F. (1970) Insects of Craters of the Moon National Monument Idaho. Volume 8. Miscellaneous Series. University of Idaho, College of Agriculture, $118 \mathrm{pp}$.

Hough, G. N. (1898a) A third American species of Cynomyia. Entomological News, 9, 165-166.

Hough, G. N. (1898b) Two new American species of Cynomyia, a study in chaetotaxy. Entomological news. 9, 105-111.

Hough, G. N. (1898c) The Muscidae collected by Dr. A. Donaldson Smith in Somaliland. Proceedings of the Academy of Natural Sciences of Philadelphia, 50, 165-187.

Hough, G. N. (1899a) Synopsis of the Calliphorinae of the United States. Zoological Bulletin, 2, 283-290. https://doi.org/10.2307/1535440 
Hough, G. N. (1899b) Some North American genera of the dipterous group, Calliphorinae Girschner. Entomological News, 10, 62-66.

Hough, G. N. (1900) South American Muscidae in the collection of S. W. Williston. The Kansas University Quarterly, 9, 203-232.

Ibáñez-Bernal, S. (2017) Actualización del Catálogo de Autoridad Taxonómica del orden Diptera (Insecta) de México. Base de datos SNIB-CONABIO, JE006. México, Cd. México. Available online at: http://www.conabio.gob.mx/institucion/proyectos/resultados/InfJE006.pdf

International Commission on Zoological Nomenclature (1992) [Opinion 1670] Calliphora vicina Robineau-Desvoidy, 1830 (Insecta, Diptera): specific name conserved. Bulletin of Zoological Nomenclature, 49, 138. https://www.tandfonline.com/doi/pdf/10.1080/03946975.1992.10539186

Jaennicke, J. F. (1867) Neue exotische Dipteren. Abhandlungen der Senckenbergischen Naturforschenden Gesellschaft, 6, 311-408.

James, M. T. (1953) Notes on the distribution, systematic position, and variation of some Calliphorinae, with particular reference to the species of western North America (Diptera, Calliphoridae). Proceedings of the Entomological Society of Washington, 55, 143-148.

James, M. T. (1955) The blowflies of California. Bulletin of the California Insect Survey, 40, 1-34.

James, M. T. (1966) The blow flies of the Galapagos Islands (Diptera: Calliphoridae). Proceedings of the California Academy of Sciences, 34, 475-482.

James, M. T. (1970) Family Calliphoridae, pp. 1-28. In: N. Papavero (Ed.). A Catalogue of the Diptera of Americas south of the United States. Museu de Zoologia, Universidade de São Paulo.

James, M. T. (1971) Two new species of Phaenicia from the West Indies (Diptera: Calliphoridae). Proceedings of the Entomological Society of Washington, 73, 381-385.

Kirby, W. (1837) Part IV Insecta, Pp: 2-325. In: J. Richardson (Ed.). Fauna Boreali-Americana, or the zoology of the northern parts of British America Volume 4. Longman, London, 700 pp. https://doi.org/10.5962/bhl.title.39293

Kosmann, C., Mello, R. de P., Harterreiten-Souza, E. S., Pujol-Luz, J. R. (2013) A list of current valid blow fly names (Diptera: Calliphoridae) in the Americas south of Mexico with key to Brazilian species. EntomoBrasilis, 6, 74-85. https://doi.org/10.12741/ebrasilis.v6i1.266

Kurahashi, H. (1991) Blow flies from Samoa with description of a new species of Chrysomya (Diptera, Calliphoridae). Japanese Journal of Entomology, 59, 627-636.

Kutty, S. N., Pape, T., Wiegmann, B. M., Meier, R. (2010) Molecular phylogeny of the Calyptratae (Diptera: Chyclorrhapha) with an emphasis on the superfamily Oestroidea and the position of Mystacinobiidae and McAlpine's fly. Systematic Entomology, 25, 614-635. https://doi.org/10.1111/j.1365-3113.2010.00536.x

Lahille, F. (1915) Nota sobre la "ura" y otras larvas dañinas de dípteros. Boletín de la Dirección General de Ganadería, Minería y Agricultura, Buenos Aires, 1-18.

Linnaeus, C. (1758) Systema naturae per regna tria naturae, secundum classes, ordines, genera, species, cum characteribus differentiis, synonymis, locis. 10th edition. Vol. 1. L. Salvii, Holmiae [= Stockholm], 824 pp.

https://doi.org/10.5962/bhl.title.542

Linnaeus, C. (1761) Fauna Svecica sistens Animalia Sveciae regni: Mammalia, Aves, Amphibia, Pisces, Insecta, Vermes. Distributa per classes \& ordines, genera \& species, cum differentiis specierum, synonymis auctorum, nominibus incolarum, locis natalium, descriptionibus insectorum. Editio altera, auctior. Laurentii Salvii, Stockholmiae [= Stockholm], [49] + 578 pp. + 2 pls. https://doi.org/10.5962/bhl.title.34906

Lioy, P. (1864) I ditteri distribuiti secondo un nuovo metodo di classificazione naturale [part]. Atti del Reale Istituto Veneto di Scienze, Lettere ed Arti, (Series 3) 9, 879-910.

Lloyd, H. (1922) Larvae of Phormia chrysorrhea [sic] Macq. found upon nestling Bluebirds. Canadian Field Naturalist, 36 (6), 1-116. 
Lopes, H. S., Albuquerque, D. O. (1955) Los insectos de las islas Juan Fernandez. 22. Calliphoridae et Sarcophagidae (Diptera). Revista Chilena de Entomología, 4, 95-119.

Mac-Lean, M., González, C. R. (2006) Catálogo de los Calliphoridae de Chile (Diptera: Oestroidea). Acta Entomológica Chilena, 30, 15-22.

Macquart, P. J. M. (1834) Insectes diptères du nord de la France. Athéricères: créophiles, oestrides, myopaires, conopsaires, scénopiniens, céphalopsides. Memoires de la Société Royale des Sciences, de l'Agriculture et des Arts à Lille, 1833, 137-368.

Macquart, P. J. M. (1843) Diptères exotiques nouveaux ou peu connus. Memoires de la Société Royale des Sciences, de l'Agriculture et des Arts à Lille, 1842, 162-460.

Macquart, P. J. M. (1846) Dipteres exotiques nouveaux ou peu connus. Supplément. Memoires de la Société (Royale) des Sciences, de l'Agriculture et des Arts à Lille, 1844, 133-364.

Macquart, P. J. M. (1847) Dipteres exotiques nouveaux ou peu connus. 2.e supplément. Memoires de la Société (Royale) des Sciences, de l'Agriculture et des Arts à Lille, 1846, 21-120.

Macquart, P. J. M. (1848) Dipteres exotiques nouveaux ou peu connus. Suite du 2.me supplément. Memoires de la Société (Royale) des Sciences, de l'Agriculture et des Arts à Lille, 1847, 161-237.

Macquart, P. J. M. (1851) Diptères exotiques nouveaux ou peu connus. Suite du 4.e supplément publié dans les Mémoires de 1849. Mémoires de la Société Royale des Sciences, de l'Agriculture et des Arts de Lille, 1850, 134-294.

Maes, J. M., Peris, S. V., González-Mora, M. D. (1994) Catálogo de los Calliphoridae (Diptera) de Nicaragua. Revista Nicaragüense de Entomología, 29, 15-24.

Malloch, J. R. (1927) Notes on Australian Diptera. No. XI. Proceedings of the Linnaean Society of New South Wales, 52, 299-335.

Malloch, J. R. (1930) Insects of Samoa and other Samoan terrestrial Arthropoda. Part VI, Diptera. Fasc. 5. British Museum (Natural History), London, 215-237.

Mañas-Jordá, S., León-Cortés, J. L., García-García, M. D., Caballero, U., Infante, F. (2017) Dipteran diversity and ecological succession on dead pigs in contrasting mountain habitats of Chiapas, Mexico. Journal of Medical Entomology, 55, 59-68. https://doi.org/10.1093/jme/tjx 190

Mariluis, J. C. (1980) Contribución al conocimiento del género Hemilucilia Brauer, 1895 (Calliphoridae, Chrysomyiinae, Hemiluciliini). Revista de la Sociedad Entomológica Argentina, 39, 83-87.

Mariluis, J. C., Mulieri, P. R. (2003) The Distribution of the Calliphoridae in Argentina (Diptera). Revista de la Sociedad de Entomología de Argentina, 62, 85-97.

Marinho, M. A. T., Junqueira, A. C. M., Paulo, D. F., Esposito, M. C., Villet, M. H., Azeredo-Espin, A. M. L. (2012) Molecular phylogenetics of Oestroidea (Diptera: Calyptratae) with emphasis on Calliphoridae: insights into the inter-familial relationships and additional evidence for paraphyly among blowflies. Molecular Phylogenetics and Evolution, 65, 840-854. https://doi.org/10.1016/j.ympev.2012.08.007

Marinho, M. A. T., Wolff, M., Ramos-Pastrana, Y., Azeredo-Espin, A. M. L., Amorim, de S. D. (2017) The first phylogenetic study of Mesembrinellidae (Diptera: Oestroidea) based on molecular data: clades and congruence with morphological characters. Cladistics, 33, 134-152.

Martínez-Ruvalcaba, H., Jaramillo, J. F., Posdas del Río, A. F., Medina, R. I. R., Escoto, R. J., Rodríguez, V. M. L. (2009) Efecto del plaguicida organofosforado paration metílico sobre el tiempo de desarrollo de la entomofauna necrófaga en cerdos (Sus scrofa). Folia Entomológica Mexicana, 48, 141-150.

McAlpine, J. F., Peterson, B. V., Shewell, G. E., Teskey, H. J., Vockeroth, J. R., Wood, D. M. (1987) Manuel of nearctic Diptera. Vol. 2., Research Branch. Agriculture Canada, Ottawa, 668 pp.

Meigen, J. W. (1826) Systematische Beschreibung der bekannten europäischen zweiflügeligen Insekten. Fünfter Theil. Schulz-Wundermann, Hamm, 412 pp.

Mello, R. P. de (1961) Contribuição ao estudo do gênero Phaenicia (R.-D., 1830) (Diptera, Calliphoridae). Memórias do Instituto Oswaldo Cruz, 59, 259-278. https://doi.org/10.1590/s0074-02761961000300002 
Mello, R. P. de (1962) Contribuição ao estudo do gênero Calliphora R.-D., 1830 (Diptera, Calliphoridae). Memórias do Instituto Oswaldo Cruz, 60, 263-274. https://doi.org/10.1590/s0074-02761962000200011

Mello, R. P. de (1965) Contribuição ao estudo do gênero Callitrogopsis Townsend (Diptera, Calliphoridae). Studia Entomologica, 8, 435-440.

Mello, R. P. de (1967) Contribuição ao estudo dos Mesembrinellinae sul-americanos (Calliphoridae). Studia Entomologica, 10, 1-80.

Mello, R. P. de (1968) Contribuição ao estudo do gênero "Paralucilia" Brauer \& Bergenstamm, 1891 (Calliphoridae, Diptera). Revista Brasileira de Biologia, 28, 177-192.

Mello, R. P. de (1969a) Notes on Laneella brunnipes (Surcouf, 1919) (Diptera, Calliphoridae). Revista Brasileira de Biologia, 29, 243-247.

Mello, R. P. de (1969b) Contribuição ao estudo do gênero Myiolucilia Hall (Diptera, Calliphoridae). Studia Entomologica, 12, 297-316.

Mello, R. P. de (1972a) Contribuição ao estudo do gênero Sarconesia Bigot, 1857 (Diptera, Calliphoridae). Revista Brasileira de Biologia, 32, 533-537.

Mello, R. P. de (1972b) Revisão das espécies do gênero Hemilucilia Brauer, 1895 (Diptera, Calliphoridae). Revista Brasileira de Biologia, 32, 539-554.

Mello, R. P. de (1974) Nova espécie do gênero Calliphora Robineau-Desvoidy, 1830. (Diptera, Calliphoridae). Arquivos da Universidade Federal Rural do Rio de Janeiro, 4, 59-63.

Mello, R. P. de (1978) Contribuição ao conhecimento do gênero Roraimomusca Townsend, 1935 (Diptera, Calliphoridae). Revista Brasileira de Biologia, 32, 895-898.

Mello, R.P. de (1996) Revisão das espécies sul americanas de Paralucilia Brauer \& Bergenstamm (Diptera: Calliphoridae). Entomología y Vectores, 3, 137-143.

Mello, R. P. de (2003) Chave para a identifcação das formas adultas das espécies da família Calliphoridae (Diptera, Brachycera, Cyclorrhapha) encontradas no Brasil. Entomología y Vectores, 10, 255-268.

Melville, R. V. (1984) Cochliomyia Townsend, 1915 (Diptera, Calliphoridae): Proposed conservation by the suppression of Callitroga Brauer, 1883. Bulletin of Zoological Nomenclature, 707, 125-128.

Moniez, R. L. (1876) Un Diptére parasite du crapaud (Lucilia bufonivora n. sp.). Bulletin scientifique, historique et littéraire du département du Nord et des pays voisins, Lille, 8, 25-27.

Norris, K. R. (1966) Daily patterns of flight activity of blowflies (Calliphoridae: Diptera) in the Canberra District as indicated by trap catches. Australian Journal of Zoology, 14, 835-853. https://doi.org/10.1071/zo9660835

Pandelle, L. (1896) Etudes sur les Muscides de France. II e partie [concl.]. Revue d'Entomologie, 15, 1230.

Pape, T., Bickel, D., Meier, R. (2009) Diptera Diversity: Status, Challenges and Tools. Brill, Leiden, The Netherlands, $479 \mathrm{pp}$.

Pape, T., Blagoderov, V., Mostovski, M. B. (2011) Order Diptera Linnaeus, 1758. In: Z. Zhi-Zhang (Ed.). Animal biodiversity: An outline of higher-level classification and survey of taxonomic richness. Zootaxa, 3148, 1-237. https://doi.org/10.11646/zootaxa.3148.1.42

Pape, T., Evenhuis, N. L. (Eds.) (2019) BioSystematic Data-base of World Diptera, Version 10. Available online at: http://www.diptera.dk

Pape, T., Wolff, M., Amat, E. C. (2004) Los califóridos, éstridos, rinofóridos y sarcofágidos (Diptera: Calliphoridae Oestridae, Rhinophoridae, Sarcophagidae) de Colombia. Biota Colombiana, 5, 201208.

Pérez-Balam, J., Quezada-Euán, J. J. G., Alfaro-Bates, R., Medina, S., McKendrick, L., Soro, A., Paxton, R. J. (2012) The Contribution of honeybees, flies and wasps to avocado (Persea Americana) pollination in southwestern Mexico. Journal of Pollination Ecology, 8, 42-47. https://doi.org/10.26786/1920-7603(2012)6

Peus, F. (1960) Zur Kenntnis der ornithoparasitischen Phormiinen (Diptera, Calliphoridae). Deutsche Entomologische Zeitschrift, Neue Folge, 7, 193-235. 
Philippi, R. A. (1861) Beschreibung einer neuen Fliege, deren Larve in der Nase und Stirnhohle einer Frau gelebt haben. Zeitschrift für Naturwissenschaften, 17, 513-515.

Pokorny, E. (1889) IV. Beitrag zur Dipterenfauna Tirols. Verhandlungen der Zoologisch-Botanischen Gesellschaft in Wien, 39, 543-574.

Poole, R. W. (1996) Diptera. In: R. W. Poole, P. Gentili (Eds.). Nomina Insecta Nearctica. A Check List of the Insects of North America. Vol. 3. Diptera, Lepidoptera, Siphonaptera. Entomological Information Services, Rockville, 15-604.

Povolnỳ, D. (1971) Synanthropy, pp. 17-54. In: B. Greenberg (Ed.). Flies and diseases. Vol. I. Ecology, classification and biotic associations. New Jersey, Princeton University Press.

Prado e Castro, C., Szpila, L., Martínez-Sánchez, A., Rego, C., Silva, I., Serrano, A. R. M., Boieiro, M. (2016) The blowflies of the Madeira Archipelago: species diversity, distribution and identification (Diptera: Calliphoridae s.l.). Zookeys, 634, 101-123. https://doi.org/10.3897/zookeys.634.9262

Robineau-Desvoidy, J. B. (1830) Essai sur les myodaires. Mémoires présentés par divers savants à l'Académie Royale des Sciences de l'Institut de France (Sciences Mathématiques et Physiques). Paris, 813 pp. https://doi.org/10.5962/bhl.title.8552

Robineau-Desvoidy, J. B. (1853) Dipteres des environs de Paris. Famille des myopaires. Bulletin de la Société des Sciences Historiques et Naturelles de l'Yonne, 7, 83-160.

Robineau-Desvoidy, J. B. (1863) Historie naturalle des dipteres des environs de Paris. Oeuvre posthume du Dr. Robineau-Desvoidy. Publiée par les soins de sa famille, sous la direction de M. H. Monceaux. Tome second. Tome second. Masson et fils, Paris, $920 \mathrm{pp}$.

Rognes, K. (1985) Revision of the bird-parasitic blowfly genus Trypocalliphora Peus, 1960 (Diptera: Calliphoridae). Entomologica Scandinavica, 15, 371-382.

Rognes, K. (1991) Blowflies (Diptera: Calliphoridae) of Fennoscandia and Denmark. Fauna Entomologica Scandinavica, Brill, $272 \mathrm{pp}$.

Rognes, K. (1997) The Calliphoridae (Blowflies) (Diptera: Oestroidea) are not a monophyletic group. Cladistics, 13, 27-68. https://doi.org/10.1006/clad.1997.0031

Rognes, K. (1998) Family Calliphoridae, pp. 617-648. In: L. Papp, B. Darvas (Eds.). Contributions to a Manual of Palaearctic Diptera (with special reference to flies of economic importance). Volume 3: Higher Brachycera. Science Herald.

Rognes, K. (2016) The identity of Calliphora bezzii Zumpt, 1956 (Diptera, Calliphoridae). Zootaxa, 4171, 191-195. https://doi.org/10.11646/zootaxa.4171.1.12

Rohdendorf, B. B. (1926) Morphologisches studium an asseren genitalorganen der Calliphorinen (Diptera). Zoologichesky Zhurnal, 6, 83-128.

Rohdendorf, B. B. (1931) Calliphorinen-Studien IV (Dipt.). Eine neue Calliphorinen-Gattung aus Ostsibirien. Zoologischer Anzeiger, 95, 175-177.

Rondani, C. (1848) Esame di varie specie d'insetti ditteri brasiliani. Studi entomologici, 1, 63-112. https://doi.org/10.5962/bhl.title.60996

Rondani, C. (1850) Osservazioni sopra alquante specie di esapodi ditteri del Museo Torinese. Nuovi Annali delle Scienze Natural, 3, 165-197.

Rondani, C. (1875) Muscaria exotica Musei Civici januensis. Fragmentum III. Species in Insula Bonae Fortunae (Borneo), provincia Sarawak annis 1865-1868, lectae a March. J. Doria et Doct. O Beccari. Annali del Museo civico di storia naturale Giacomo Doria, 7, 421-464.

Sabrosky, C. W., Bennett, G. F., Whitworth, T. L. (1989) Bird blow flies (Protocalliphora) in North America (Diptera: Calliphoridae) with notes on Palearctic species. Smithsonian Institution Press, Washington, $328 \mathrm{pp}$.

Sabrosky, C. W. (1999) Family-group names in Diptera and Bibliography. The International Journal of North American Dipterists' Society, 10, 1-576. 
Schiner, J. I. R. (1862) Fauna Austriaca: die Fliegen (Diptera) Nach der analytischen Methode bearb., mit der Characteristik almmilicher europäischer Gattungen, der Beechraibung aller in Deutschland vorkommenden Arten und der Aufzahlung aller bisher beschriebenen europaischen Arten. Theil I. Wien: C. Gerolds Sohn, 674 pp. https://doi.org/10.5962/bhl.title.8525

Schiner, J. I. R. (1868) Diptera, Pp: 1-388. In: C. F. Felder von, B. F. Wüllerstof-Urbair von (Eds.). Reise der osterreichischen Fregatte Novara um die Erde in den Jahren 1857, 1858, 1859, unter den Befehlendes Comodore B. von Wüllerstof-Urbair. Zoologischer Theil, 9, bd. 2, ABT 1b B. K. Gerold's Sohn, Wien. https://doi.org/10.5962/bhl.title.1597

Séguy, E. (1925) Description de quatre Calliphorines nouveaux (Dipt.). Bulletin de la Société entomologique de France, 30, 303-304.

Séguy, E. (1928) Études sur les mouches parasites. Tome I. Conopides, oestrides et calliphorines de l'Europe occidentale. Recherches sur la morphologie et la distribution géographique des diptéres á la larves parasites. Encyclopédie Entomologique, 9, 1-251.

Senior-White, R. A. (1924) New and little known Oriental Tachinidae. Spolia zeylanica, 13, 103-119.

Senior-White, R., Aubertin, D., Smart, F. (1940) The fauna of British India, Diptera, 6, 1-288.

Shannon, R. C. (1923) Genera of Nearctic Calliphoridae, blowflies, with revision of the Calliphorini (Diptera). Insecutor inscitiae menstruus, 11, 101-118.

Shannon, R. C. (1924) Nearctic Calliphoridae, Luciliini (Diptera). Insecutor Inscitiae Menstruus, 12, 6781.

Shannon, R. C. (1926) Synopsis of the American Calliphoridae (Diptera). Proceedings of the Entomological Society of Washington, 28, 115-139.

Shannon, R. C., Del Ponte, E. (1926) Sinopsis parcial de los Muscoideos argentinos. Revista del Instituto Bacteriologico, 4, 549-590.

Shannon, R. C., Dobroscky, I. D. (1924) The North American bird parasites of the genus Protocalliphora (Calliphoridae, Diptera). Journal of the Washington Academy of Sciences, 14, 247-253.

Smith, F. (1876) Preliminary notice of new species of Hymenoptera, Diptera, and Forficulidae collected in the island of Rodriquez by the naturalists accompanying the Transit-of-Venus Expedition. The Annals and Magazine of Natural History; Zoology, Botany, and Geology, 17, 447-451.

Stanford-Camargo, S., Medina-Ortiz, R. G., Dávila-Vega, J. P., Domínguez-Fuentes, M. J., DuarteMartínez, H. E., Pérez-Arteaga, E., Zagal-Salinas, A. (2017) Atrópodos sarcosaprófagos asociados a tres zonas en un bosque mesófilo de montaña en Huauchinango, Puebla, México. Boletín de la Sociedad Mexicana de Enomología, 4, 93-97.

Stevens, J., Wall, R. (1996) Classification of the genus Lucilia (Diptera: Calliphoridae): a preliminary parsimony analysis. Journal of Natural History, 30, 1087-1094. https://doi.org/10.1080/00222939600770581

Surcouf, J. M. R. (1919) Revision des Muscidaes Testaceae. Nouvelles Archives du Museum D'Histoire Naturelle de Paris, Series V, 27-394.

Tantawi, T. I., Whitworth, T. (2014) First record of Lucilia bufonivora Moniez, 1876 Diptera: Calliphoridae) from North America and key to North American species of the L. bufonivora species group. Zootaxa, 3881, 101-124. http://doi.org/10.11646/zootaxa.3881.2.1

Tantawi, I. T., Whitworth, T. L., Sinclair, B. J. (2017) Revision of the Nearctic Calliphora RobineauDesvoidy (Diptera: Calliphoridae). Zootaxa, 4226, 301-347. https://doi.org/10.11646/zootaxa.4226.3.1

Thomas, H. T. (1951) Some species of the blow-fly genera Chrysomyia R.-D., Lucilia R.D., Hemipyrellia Tnsd. and Calliphora R.-D. from South-eastern Szechuan, China. Proceedings of the Zoological Society of London, 121, 147-200. https://doi.org/10.1111/j.1096-3642.1951.tb00741.x 
Thomson, C. G. (1869) Diptera. Species nova descripsit, pp. 443-614. In: Kongliga svenska fregatten Eugenies resa omkring jorden under befal af C. A. Virgin, (Zoologi) 1, Insecta. P. A. Norstedt \& Soner, Stockholm, 617 pp.

Townsend, C. H. T. (1908) The taxonomy of the muscoidean flies, including descriptions of new genera and species. Smithsonian Miscellaneous Collections, 51, 1-138.

Townsend, C. H. T. (1915a) Synonymical notes on Muscoidea. Insecutor Inscitiae Menstruus, 3, 115-122.

Townsend, C. H. T. (1915b) Proposal of new muscoid genera for old species. Proceedings of the biological society of Washington, 28, 19-23.

Townsend, C. H. T. (1915c) A new generic name for the screw-worm fly. Journal of the Washington Academy of Sciences, 5, 644-646.

Townsend, C. H. T. (1916a) New genera and species of muscoid flies. Proceedings of the United States National Museum, 51, 299-323. https://doi.org/10.5479/si.00963801.2301.541

Townsend, C. H. T. (1916b) Designations of muscoid genotypes, with new genera and species. Insecutor Inscitiae Menstruus, 4, 4-12.

Townsend, C. H. T. (1918) New muscoid genera, species and synonymy (Diptera). Insecutor Inscitiae Menstruus, 6, 151-156.

Townsend, C. H. T. (1919) New genera and species of muscoid flies. Proceedings of the United States National Museum, 56, 541-592.

Townsend, C. H. T. (1934a) Muscoid notes and descriptions. Revista de Entomología, 4, 110-112.

Townsend, C. H. T. (1934b) A New Genus of Cobboldiini and a New Genus of Chrysomyinae Flies (Dipt.: Oestroidea, Muscoidea). Entomological news, 45, 277-286.

Townsend, C. H. T. (1935) New muscoid genera, mainly from the Neotropical region. Revista de Entomología, 5, 68-74.

Townsend, C. H. T. (1937) ManuaL ot Myiology in twelve parts. Part. 5. Muscoid generic diagnoses and data. Glossinini to Agriini. São Paulo, Itaquaquecetuba, 234 pp.

Valdés-Perezgasga, M. T., García-Espinoza, F. (2014) Dinámica de califóridos (Diptera: Calliphoridae) en tres municipios de La Comarca Lagunera en el periodo de 2012-2013. Entomología Mexicana, $1,345-350$.

Valdés-Perezgasga, M. T., García-Espinoza, F., Pastrana-Ortíz, E. (2012) Morfología de tres especies de califóridos (Diptera: Calliphoridae) de importancia forense en Torreón, Coahuila. Entomología Mexicana, 11, 891-896.

Vaughan, T. W. (1903) Corrections to the nomenclature of the Eocene fossil corals of the United States. Proceedings of the Biological Society of Washington, 16, 1-101.

Verhuell, Q. M. R. (1850) Mededeeling der metamorphose van eene in Guyana voorkomende vliegensoort. Tijdschrift voor de wis-en natuurkundige wetenschappen, 3, 273-275.

Villeneuve, J. (1927) Sur Onesia genarum (Zett.) (Dipt.). Bulletin et Annales de la Société Entomologique de Belgique, 66, 1-357.

Walker, F. (1836) Descriptions, \&c. of the Diptera. In: J. Curtis, A. H. Haliday, F. Walker (Eds.), Descriptions, \&c. of the insects collected by Captain P.P. King, R.N., F.R.S., in the survey of the Straits of Magellan. Transactions of the Linnaean Society of London, 17, 331-359.

Walker, F. (1849) List of the specimens of dipterous insects in the collection of the British Museum. Part II, 231-484; Part III: 85-687; Part IV: 689-1172, British Museum (Natural History), London.

Walker, F. (1852) Diptera. Part III, pp. 157-252. In: W. W. Saunders (Ed.). Insecta Saundersiana: or characters of undescribed insects in the collection of William Wilson Saunders, Esq., F.R.S., F.L.S., \& $C$. Vol. 1. Van Voorst, London, 474 pp.

Walker, F. (1853) Diptera. Part IV, pp. 253-474. In: W. W. Saunders (Ed.). Insecta Saundersiana: or characters of undescribed insects in the collection of William Wilson Saunders, Esq., F.R.S., F.L.S., $\& C$. Vol. 1. Van Voorst, London, 474 pp.

Walker, F. (1861) Characters of undescribed Diptera in the collection of W. W. Saunders, Esq., F. R. S. \& C. Transactions of the Entomological Society of London, 5, 268-296. 
Waterhouse, D. F., Paramonov, S. J. (1950) The status of the two species of Lucilia (Diptera, Calliphoridae) attacking sheep in Australia. Australian Journal of Scientific Research, 3, 310-336. https://doi.org/10.1071/bi9500310

Whitworth, T. L. (2006) Keys to the genera and species of blow flies (Diptera: Calliphoridae) of America north of Mexico. Proceedings of the Entomological Society of Washington, 108 (3), 689-725.

Whitworth, T. L. (2010) Keys to the blow fly species (Diptera Calliphoridae) of America, north of Mexico, pp. 581-625. In: J. H. Byrd, J. L. Castner (Eds.). Forensic Entomology, the utility of arthropods in legal investigations. CRC Press, Boca Raton.

Whitworth, T. L. (2012) Identification of Neotropical blow flies of the genus Calliphora RobineauDesvoidy (Diptera: Calliphoridae) with the description of a new species. Zootaxa, 3209, 1-27. http://dx.doi.org/10.11646/zootaxa.3209.1.1

Whitworth, T. L. (2014) A revision of the Neotropical species of Lucilia Robineau-Desvoidy (Diptera: Calliphoridae). Zootaxa, 3810, 1-76. https://doi.org/10.11646/zootaxa.3810.1.1

Wiedemann, C. R. W. (1819) Brazilianische Zweiflügler. Zoologisches Magazin, 1, 40-56.

Wiedemann, C. R. W. (1830) Aussereuropaische zweiflugelige Insekten. Zweiter Theil, Schulz, Hamm, $684 \mathrm{pp}$.

Wolff, M. (2013) A new species of Mesembrinella (Diptera: Calliphoridae: Mesembrinellinae) from Colombia. Revista Colombiana de Entomología, 39, 120-124.

Wolff, M., Kossmann, C. (2016) Families Calliphoridae and Mesembrinellidae. Zootaxa, 4122, 856-875. http://dx.doi.org/10.11646/zootaxa.4122.1.72

Wulp, F. M. van der (1896) Diptera, Pp: 1-294. In: F. D. Godman, O. Salvin (Eds.). Biologia CentraliAmericana. Zoologia Insecta-Diptera. Vol. 2. Taylor \& Francis, London, 514 pp.

Zetterstedt, J. W. (1838) Sectio tertia. Diptera. Dipterologis Scandinaviae amicis et popularibus carissimus, Pp: 477-868. In: J. W. Zetterstedt (Ed.). Insecta Lapponica. L. Voss, Lipsiae [= Leipzig].

Zumpt, F. (1956) Calliphorinae, 1-48. In: E. Lindner (Ed.). Die Fliegen der Palaearktischen Region. Schweizerbart, Stuttgart.

Zumpt, F. (1965) Myiasis in man animals of the Old World. A textbook for physicians, veterinarians and zoologists. Butterworths, London, 267 pp. 